\title{
Comparación de los resultados de la refracción subjetiva con dos métodos diferentes
}

\author{
Córdova-Castellanos María Fernanda*, Amézquita-Moreno Miguel Ángel*, Ramírez-Espinosa \\ Gilberto Enrique*, Ruano-González Lorena*, Salas-Hernández Luis Héctor**, Casillas-Casillas \\ Elizabeth**.
}

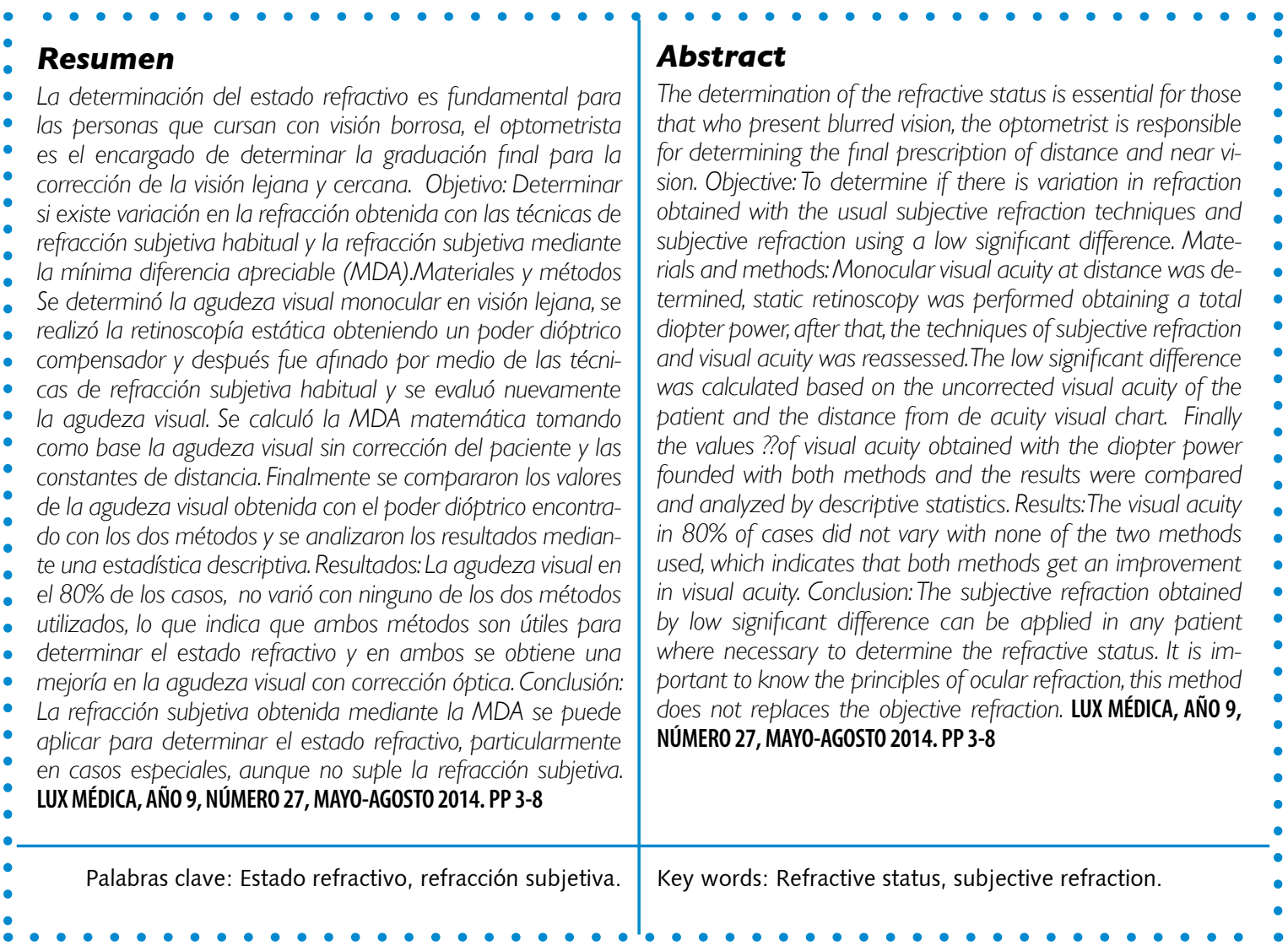

\footnotetext{
* Estudiantes del noveno semestre de la Licenciatura de Optometría del Centro de Ciencias de la Salud de la Universidad Autónoma de Aguascalientes.

** Profesor investigador del Departamento de Optometría del Centro de Ciencias de la Salud de la Universidad Autónoma de Aguascalientes
}

Fecha de recibido: 3 de marzo de 2014

Fecha de aceptado: 3 de mayo de 2014

Correspondencia: MCB Luis Héctor Salas Hernández. Unidad Médica Didáctica, edificio 101, planta alta. Avenida Universidad \#940 CP20131. Aguascalientes., Ags., México. Teléfono 01(449)9108441. Correo electrónico Ihsalas@correo.uaa.mx 


\section{Introducción}

La determinación del estado refractivo es fundamental para las personas que padecen de visión borrosa, el optometrista es el encargado de determinar la graduación final para la corrección de la visión lejana y cercana. ${ }^{1}$ Esta graduación puede ser indicada en anteojos o lentes de contacto, realizando la refracción objetiva y subjetiva, de ahí la importancia de un buen examen visual optométrico. ${ }^{2}$ La queratometría y la retinoscopía estática forman parte de la refracción objetiva. La primera es un procedimiento donde se miden 3 $\mathrm{mm}$ centrales de la superficie anterior de la córnea. La segunda tiene como propósito determinar el estado refractivo mientras el paciente observa un objeto situado a seis metros con su acomodación relajada.

La refracción subjetiva tiene como propósito determinar por medios subjetivos, la combinación de lentes esféricas o cilíndricas necesarias para situar artificialmente el punto remoto de cada uno de los ojos en el infinito. Esta combinación de lentes proporciona la mejor agudeza visual posible con la acomodación relajada. Para garantizar que la acomodación se encuentre relajada se debe miopizar el sistema visual colocando lentes positivas. ${ }^{3,4}$ Una vez realizada la refracción objetiva se procede a realizar la refracción subjetiva habitual. ${ }^{5-7}$ (tabla 1)

\section{Tabla |}

\section{Pruebas de refracción subjetiva}

\begin{tabular}{|ll}
\hline Máximo positivo con su máxima agudeza visual & Afinación de poder esférico \\
\hline Bicromática o test Duocromo & Afinación de poder esférico \\
\hline Reloj astigmático & Afinación de eje y poder cilíndrico \\
\hline Cilindro cruzado de Jackson (CCJ) & Afinación de eje y poder cilíndrico \\
\hline
\end{tabular}

Otra prueba no muy habitual e inclusive desconocida para algunos optometristas que ayuda a determinar el estado refractivo de manera subjetiva es el cálculo de la mínima diferencia apreciable (MDA). ${ }^{7}$ Esta prueba es aplicada en pacientes que cursan con baja visión, pero no es exclusiva para este tipo de pacientes, se puede realizar en pacientes que no cursen con baja visión, pues se puede determinar el estado refractivo sin realizar la retinoscopía estática. Las causas por las que en ocasiones no se pude realizar la retinoscopía en pacientes son: leucomas corneales, cataratas, opacidades en los medios refringentes, degeneraciones corneales o en pacientes donde simplemente no se observa reflejo del retinoscopio, ${ }^{8}$ en algunos casos estos padecimientos oculares pueden ocasionar visión baja.

Para obtener la MDA se debe realizar la medición de la agudeza y se tomará en cuenta la distancia a la cual se realiza la prueba, se utiliza la siguiente fórmula y constantes para determinar la MDA matemática. ${ }^{9}$ (tabla 2) 


\section{Tabla 2}

Fórmula para calcular la MDA matemática

\begin{tabular}{|c|c|c|}
\hline \multirow{4}{*}{$M D A=$} & \multirow{2}{*}{ DENOMINADOR DE SNELLEN } & $6 \mathrm{mts}=30$ \\
\hline & & $5 \mathrm{mts}=25$ \\
\hline & \multirow{4}{*}{ poder esférico y cilíndrico inicial } & $4 \mathrm{mts}=20$ \\
\hline & & $3 \mathrm{mts}=15$ \\
\hline \multirow{2}{*}{ Este resultado será el poder esférico y cilíndrico inicial } & & $2 \mathrm{mts}=10$ \\
\hline & & $1 \mathrm{mts}=0.5$ \\
\hline
\end{tabular}

Una vez obtenida la MDA matemática, hay que tomar en cuenta que este resultado será nuestro poder esférico y cilíndrico inicial, posteriormente, verificando la preferencia del paciente hacia lentes positivas o negativas y para finalizar, colocando al armazón de prueba el poder de la MDA matemática según la respuesta del paciente, anteponer lentes esféricas con poder de \pm 1.00 . Esto aumentará o disminuirá el poder de la MDA matemática y de esta manera se afina el poder esférico después el eje y finalmente cilindro. Se debe de tomar en cuenta que a peor agudeza visual mayor será la $\mathrm{MDA}^{8}$.

El objetivo de este trabajo fue determinar si existe variación en la refracción obtenida con las técnicas de refracción objetiva y subjetiva habitual y la refracción subjetiva mediante el cálculo y afinación de la mínima diferencia apreciable (MDA).

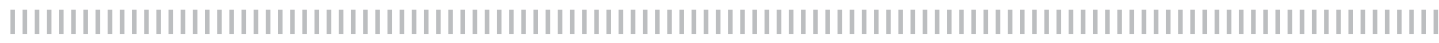

\section{Material y métodos}

Se realizó un estudio observacional, comparativo, prospectivo con un tamaño de muestra de 100 ojos, género indistinto, de edades entre 17 a 35 años. Se excluyeron los casos con alguna patología ocular y personas con problemas acomodativos. Se determinó la agudeza visual monocular en visión lejana y cercana, se realizó la retinoscopía estática obteniendo un poder dióptrico que después fue afinado por medio de las técnicas de la refracción subjetiva habitual y se evaluó nuevamente la agudeza visual para observar la mejoría con anteojos. Después se llevó a cabo el cálculo de la MDA matemática, afinando el poder esférico, eje y cilindro. Una vez obtenidos los valores dióptricos y de agudeza visual lograda, se compararon los resultados entre la refracción objetiva y subjetiva habitual y la MDA y se analizaron los resultados mediante una estadística descriptiva. Como parte del análisis descriptivo se construyó una tabla de frecuencias para la interpretación de los datos.

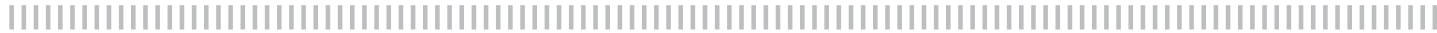

\section{Resultados}

Nuestros resultados mostraron que en el $80 \%$ de los casos, la agudeza visual no varió con ninguno de los dos métodos utiliza- dos, lo que nos indica que ambos métodos brindan una buena agudeza visual con la corrección óptica encontrada en refracción subjetiva habitual y la MDA. (figura 1) 


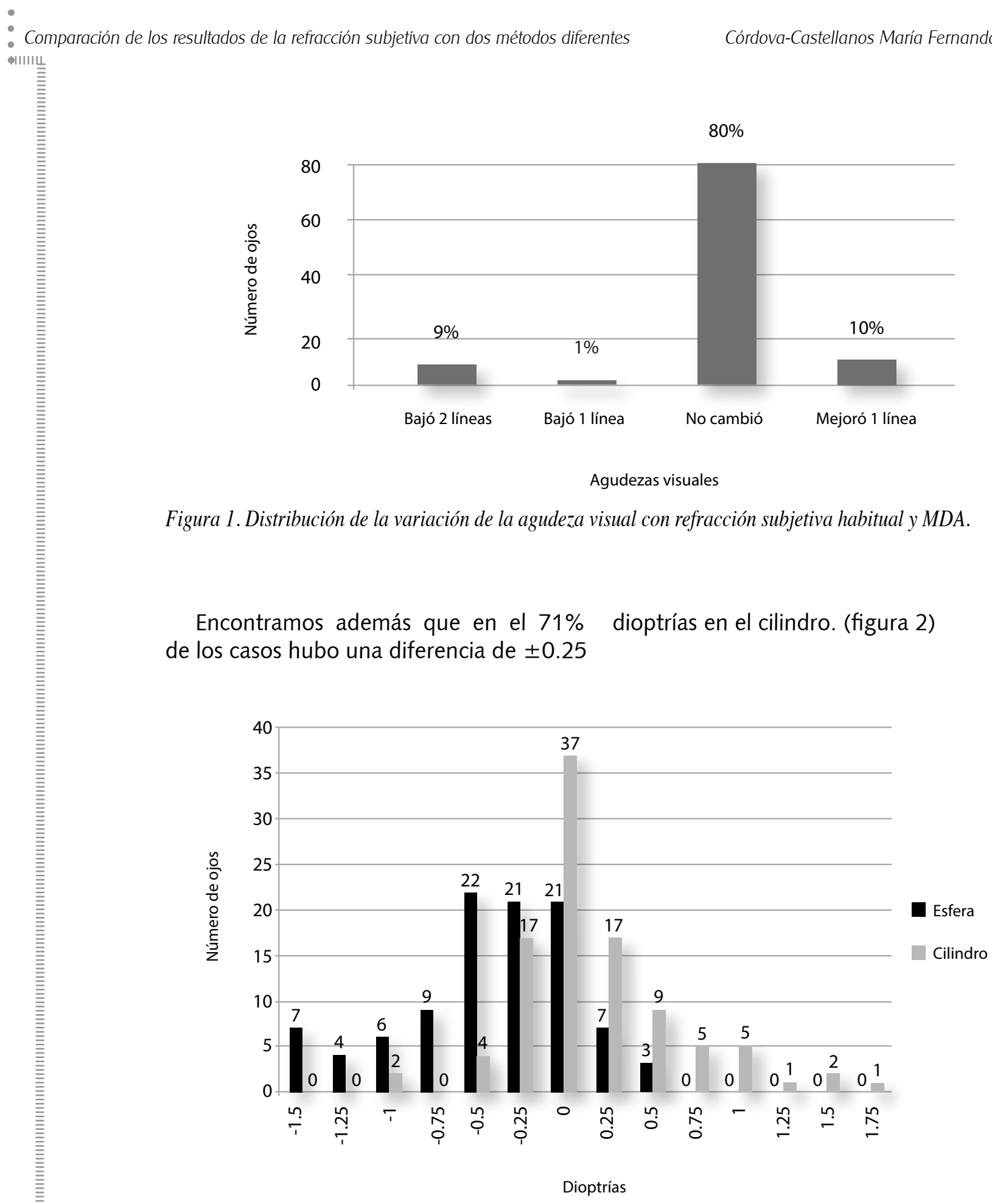

Figura 2. Distribución de las variaciones en poder esférico y cilíndrico.

En el $62 \%$ de los casos el eje osciló entre dose los porcentajes restantes en resulta$0^{\circ}$ a $5^{\circ}$ de diferencia (figura 3 ), distribuyén- dos que no son clínicamente significativos. 


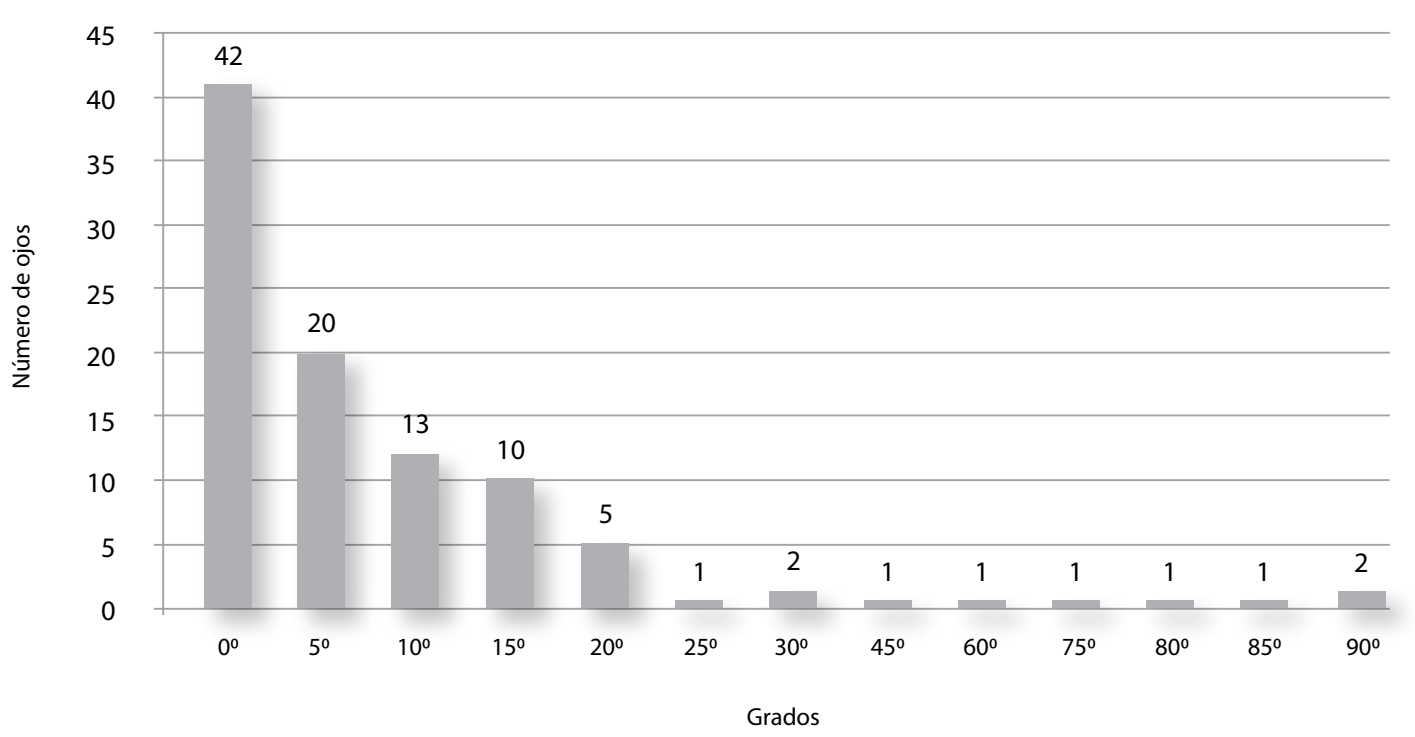

Figura 3. Distribución de las variaciones en eje corrector

\section{Discusión}

La distribución mundial de las principales causas de deficiencia visual son los errores de refracción (miopía, hipermetropía o astigmatismo) no corregidos $43 \%$. Las cataratas no operadas $33 \%$ y glaucoma $2 \%$. Aproximadamente un $90 \%$ de la carga mundial de discapacidad visual se concentra en los países en desarrollo.

Alrededor de un $65 \%$ de las personas con discapacidad visual son mayores de 50 años, si bien este grupo de edad apenas representa un $20 \%$ de la población mundial. Con el creciente envejecimiento de la población en muchos países, aumentará también el número de personas que corren el riesgo de padecer discapacidades visuales asociadas a la edad. 9, 10

Se estima que el número de niños con deficiencia visual asciende a 19 millones, de los cuales 12 millones la padecen debido a errores de refracción, fácilmente diagnosticables y corregibles. Unos 1.4 millones de menores de 15 años sufren ceguera irreversible. En México más del $40 \%$ de la población padecen algún tipo de ametropía. ${ }^{11}$

Queirós, Hopkins y Twelker trataron de comparar los resultados de técnicas como: la retinoscopía de Mohindra, autorefractometro, la retinoscopía con lente de $+2.00 \mathrm{D}$ y bajo cicloplegía entre otros. Sin embargo ninguna trató de comparar los resultados entre la retinoscopía subjetiva y la MDA. ${ }^{12-14}$

Frannin, Llewellyn y Borras, el paciente utiliza su acomodación para enfocar la imagen borrosa en retina; la media de la esfera es $\pm 0.50 \mathrm{D}$ predominando en el $69 \%$ de los casos los valores negativos, esto indica que el paciente utiliza su acomodación al realizar la MDA. ${ }^{15-17}$

La MDA es el método subjetivo más utilizado en pacientes de baja visión pero no es exclusivo para este tipo de pacientes, se puede realizar en cualquier paciente cuando se requiera determinar el estado refractivo por alguna condición que impida llevar a cabo la retinoscopía. ${ }^{18}$

Una característica importante a tomar en cuenta de la refracción subjetiva colocar el poder positivo con el que se miopiza al paciente con un poder dióptrico suficiente para que el paciente aprecie los cambios en lo borroso.

Se debe de usar el poder adecuado en los cilindros cruzados de Jackson para exa- 
minar astigmatismos precisando el alineamiento de los ejes y las líneas del poder.

Con frecuencia son necesarias las repeticiones en el cambio de lente para verificar las respuestas subjetivas del paciente. ${ }^{19,20}$

Tanto la refracción subjetiva como la MDA son procedimientos aceptables para determinar el estado refractivo, ya que la diferencia entre ellos según los resultados de este estudio en esfera es de $\pm 0.50 \mathrm{D}$, no hay diferencia en el poder cilíndrico ni en el eje y se obtiene la misma agudeza visual.

\section{Conclusiones}

La toma de decisiones clínicas en optometría depende con frecuencia de la precisión de la determinación del estado refractivo y de la mejor agudeza visual. La elección correcta de una o varias técnicas hace que el examen optométrico sea exitoso, obteniendo la mejor agudeza visual del paciente.

La técnica de la MDA es una opción útil para determinar el estado refractivo en condiciones donde no se pueda realizar la retinoscopía subjetiva convencional, proporcionando al optometrista una herramienta más para desempeñar su trabajo con calidad.

\section{Bibliografía}

1. Bharadwaj SR, Malavita M, Jayaraj J. A psychophysical technique for estimating the accuracy and precision of retinoscopy. Clin Exp Optom. 2014 Mar;97(2):164-70.

2. Hopkins $S$, Sampson GP, Hendicott $P$, Lacherez $P$, Wood JM. Refraction in children: a comparison of two methods of accommodation control. Optom Vis Sci. 2012 Dec;89(12):1734-9.

3. Lam N, Leat SJ. Barriers to accessing low-vision care: the patient's perspective. Can J Ophthalmol. 2013 Dec;48(6):458-62.

4. Pearce $E$, Crossland MD, Rubin GS. The efficacy of low vision device training in a hospital-based low vision clinic. Br J Ophthalmol. 2011 Jan;95(1):105-8.

5. Bradley A, Xu R, Thibos L, Marin G, Hernandez M. Influence of spherical aberration, stimulus spatial frequency, and pupil apodisation on subjective refractions. Ophthalmic Physiol Opt. 2014 Jan 7.

6. Vilaseca $M$, Arjona $M$, Pujol J, Peris E, Martínez V. Non-cycloplegic spherical equivalent refraction in adults: comparison of the double-pass system, retinoscopy, subjective refraction and a table-mounted autorefractor. Int J Ophthalmol. 2013 Oct $18 ; 6(5): 618-25$

7. Nemeth G, Lipecz A, Szalai E, Berta A, Modis L Jr. Accommodation in phakic and pseudophakic eyes measured with subjective and objective methods. J Cataract Refract Surg. 2013 Oct;39(10):1534-42. 8. Barañano Ángel, Formación en la Baja Visión. Madrid España. Edit AVS 2011; 1(10):12-16.

9. Organización Panamericana de Salud, Uruguay 2013 http://www.paho.org/uru/index php?option $=$ com_content $\&$ vie $=$ article \&id $=770:$ ceg uera-y-discapacidad-visual\&catid=704:discapacidad accesibilidad\&itemid $=247$

10. Eleanor E. Faye y otros. Manual de entrenamiento en la atención de la baja visión. EU. Lighthouse. 2003; 1(2): 24-30.

11. http://www.encuentra.gob.mx/resultsAPF. $\mathrm{html}$ ?q= defectos\%20visuales\&client=salud Secretaria de Salud México.

12. Antonio Queirós, et al. Optometry and vision science. University of Minho, University of Santiago de Compostela, Spain vol. 82, no. 1(23):. 64-68.

13. Shelley Hopkins Et. Optometry and vision science. EU Vol. 89, no. 1(12): 1734-1739.

14. J. Daniel Twelker, et al. Optometry and vision science. University of California, school of optometry, Berkeley, California. 2009 vol. 78, no. 4(2): 215-222.

15. Tray E, Frannin. Óptica clínica. Ediciones omega. Segunda España 1996 edición. Vol 1(3): 123-130.

16. Keith Edward, Richard LLewellyn. Optometry. Masson, SA. EU. 1993, 1(18):166- 173.

17. M. Rosa Borras García, Juan Gispets Parcerisas. Visión binocular diagnóstico y tratamiento. Alfa omega. España 2000,1(9):49-64.

18. Mayo,Perla Catherine. Inducción a la Baja visión. Edit Bastón Verde. Argentina 2011; 5(21): 26-45.

19. Eleanor E. Faye y otros. Manual de entrenamiento en la atención de la baja visión. Lighthouse.EU. 2003; 3(32): 124-129.

20. Jane Macnaughton. Evaluación en la Baja Visión. MASSON. Barcelona España-Reino Unido. 2006; 2(41): 65-67. 